\title{
On a Special Quaternionic Sequence
}

\author{
Şule Çürük, Serpıl Halici* \\ Department of Math., Science and Arts Faculty, Pamukkale University, Pamukkale Denizli, Turkey
}

Copyright (C) 2018 by authors, all rights reserved. Authors agree that this article remains permanently open access under the terms of the Creative Commons Attribution License 4.0 International License

\begin{abstract}
In this study, we investigate Fibonacci quaternions and their some important properties. Then, we define a special sequence using the elements of the Fibonacci quaternion sequence. Furthermore, we calculate the autocorrelation, right and left periodic autocorrelation values by using the elements of the newly defined sequence.
\end{abstract}

Keywords Recurrence Relation, Special Sequences, Quaternions

\section{INTRODUCTION}

Quaternions are defined by W. R. Hamilton as a generalization of complex numbers[3]. The author discovered quaternions in his work that begin by asking the question "If there is a turn in 2-dimensional spaces, it also exists in 3-dimensional spaces" during the $19 t h$ century. In the literature, the set of this numbers is denoted by the letter $\mathbb{H}$ in the memory of Hamilton.

Any real quaternion number $q$ is written in the form below.

$$
q=a_{0}+a_{1} i+a_{2} j+a_{3} k ; a_{0}, a_{1}, a_{2}, a_{3} \in \mathbb{R}
$$

Here $i, j, k$ are imaginary units. $\mathbb{H}$ is a vector space and the set of $\{1, i, j, k\}$ is a base of this space. While the addition on $\mathbb{H}$ is the component to component, the multiplication is performed taking into account the following equalities[10].

$$
i j=-j i=k, \quad j k=-k j=i, \quad k i=-i k=j
$$

and

$$
i^{2}=j^{2}=k^{2}=i j k=-1 .
$$

Taking the rules (1.2) and (1.3) into consideration, the following table can be construct.

\begin{tabular}{|c|c|c|c|c|}
\hline & 1 & $i$ & $j$ & $k$ \\
\hline 1 & 1 & $i$ & $j$ & $k$ \\
\hline$i$ & $i$ & -1 & $k$ & $-j$ \\
\hline$j$ & $j$ & $-k$ & -1 & $i$ \\
\hline$k$ & $k$ & $j$ & $-i$ & -1 \\
\hline
\end{tabular}

Table 1. The multiplication of bases elements

The set $\mathbb{H}$ is a skew-field and an associative division algebra according to this multiplication. The element $q$ can be written as vector and scalar parts $[4,10]$;

$$
q=a_{0}+v
$$

where $v=a_{1} i+a_{2} j+a_{3} k$ is the vector part of $q$ and $a_{0}, a_{1}, a_{2}, a_{3}$ are real components. $\mathbb{H}$ is a vector space on $\mathbb{R}$. Moreover, $\mathbb{H}$ is a algebra generated by the elements $i$ and $j$ on the real field. Involution process is also commonly known as conjugate operation $[2,3,4,9]$.

$$
:: \bar{i}=-i, \quad \bar{j}=-j, \quad \overline{(i j)}=\bar{j} \bar{i}=-i j .
$$


For any quaternion $q$, the conjugate operation is defined as $\bar{q}=a_{0}-a_{1} i-a_{2} j-a_{3} k$. For the quaternions $p$ and $q$, the properties of this operation can be given as

$$
\overline{\bar{q}}=q, \quad \overline{q+p}=\bar{q}+\bar{p}, \quad \overline{q p}=\bar{p} \bar{q} .
$$

The norm of quaternion $q$ is defined as follows;

$$
|q|=\sqrt{q \bar{q}}=\sqrt{\bar{q} q}=\sqrt{a_{0}^{2}+a_{1}^{2}+a_{2}^{2}+a_{3}^{2}} .
$$

According to the function in (1.4), $q \neq 0, q$ can be reversed in the set $\mathbb{H}$, that is

$$
q^{-1}=\frac{\bar{q}}{\|q\|}
$$

Different quaternion sequences which are their coefficients were taken from some special sequences were studied in the literature. The first of these studies belongs to A. F. Horadam. Horadam studied different quaternion sequences taken from some special sequences. He defined the Fibonacci and Lucas quaternions as shown in the equation (1.8) and (1.9) below, respectively[4].

$$
Q_{n}=F_{n}+F_{n+1} i+F_{n+2} j+F_{n+3} k
$$

and

$$
K_{n}=L_{n}+L_{n+1} i+L_{n+2} j+L_{n+3} k .
$$

And he called as Fibonacci and Lucas quaternions. In addition, the author also defined the conjugate and norm of quaternion $Q_{n}$ as

$$
\overline{Q_{n}}=F_{n}-F_{n+1} i-F_{n+2} j-F_{n+3} k
$$

and

$$
\left|Q_{n}\right|=\sqrt{Q_{n} \overline{Q_{n}}}=\sqrt{\overline{Q_{n}} Q_{n}}=\sqrt{F_{n}^{2}+F_{n+1}^{2}+F_{n+2}{ }^{2}+F_{n+3}^{2}}
$$

respectively. The formulas (1.8) and (1.9) give the $n t h$ term of Fibonacci and Lucas quaternions, respectively. It should be noted that every Fibonacci number $F_{n}$, except the first two, is the sum of its two immediate predecessors, $F_{n-1}$ and $F_{n-2}$. Accordingly, for $n \in \mathbb{Z}, n \geq 2$ and the initial conditions $F_{0}=0, F_{1}=1$ this sequence is written as follows[6, 12]

$$
F_{n}=F_{n-1}+F_{n-2} \text {. }
$$

Now, let's define a new set of elements consisting of Fibonacci quaternions: if we denote the sequence which its $n$ terms is $Q_{n}$ like

$$
\left\{Q_{n}\right\}_{n \geq 0}=\left\{Q_{0}, Q_{1}, Q_{2}, \ldots, Q_{n}, \ldots\right\},
$$

then the general term of this sequence is given by the following formula.

$$
Q_{n}=\frac{1}{\sqrt{5}}\left(\underline{\alpha} \alpha^{n}-\underline{\beta} \beta^{n}\right) .
$$

Note that the formula (1.14) is obtained by Halici in[2]. Here, $\alpha$ and $\beta$ are the roots of the characteristic equation of the sequence $\left\{F_{n}\right\}_{n \geq 0}$ and the values $\underline{\alpha}$ and $\underline{\beta}$ are follows

$$
\underline{\alpha}=1+i \alpha+j \alpha^{2}+k \alpha^{3}, \underline{\beta}=1+i \beta+j \beta^{2}+k \beta^{3} .
$$

\section{A PERIODIC SEQUENCE WITH FIBONACCI COEFFICIENTS}

In this section, we first introduce some concepts and notations. By using the definition of norm of a quaternion, let us define the norm of sequence $\left\{Q_{n}\right\}$ as

$$
\left\|\left\{Q_{n}\right\}_{n \geq 0}\right\|=\left\|Q_{0}, Q_{1}, Q_{2}, \ldots, Q_{n}, \ldots\right\|=\sum_{t=0}^{n-1}\left\|Q_{t}\right\| .
$$

Now, let's display the following sequence as $P_{a b}$,

$$
P_{a b}=\left\{a, b, \frac{b+1}{a}, \frac{a+b+1}{a b}, \frac{a+1}{b}, a, b, \ldots\right\}
$$


where $a$ and $b$ are real numbers. Then, using the sequence (2.2), let's define also a new sequence $U_{n}$ as follows.

$$
U_{n+1}=\frac{1+U_{n}}{U_{n-1}}, \quad U_{0}=a, \quad U_{1}=b \quad n \geq 1 .
$$

So, let's define a new quaternion sequence using the elements of the Fibonacci quaternion sequence and denote it with $\left\{Q_{n}\right\}_{n} \geq 0$ . For $n \geq 2$, the $n t h$ term of the sequence $\left\{Q_{n}\right\}_{n \geq 0}$ is

$$
Q_{n}=\frac{1+Q_{n-1}}{Q_{n-2}}
$$

If we calculate the initial values, then we have $Q_{0}=i+j+2 k, Q_{1}=1+i+2 j+3 k$. Thus, the elements of $\left\{Q_{n}\right\}_{n \geq 0}$ are

$$
\left\{Q_{n}\right\}_{n \geq 0}=\left\{Q_{0}, Q_{1}, \frac{Q_{1}+1}{Q_{0}}, \frac{Q_{0}+Q_{1}+1}{Q_{0} Q_{1}}, \frac{Q_{0}+1}{Q_{1}}, \ldots\right\} .
$$

Thus, we get $Q_{t}=Q_{t+5}$, that is the sequence $\left\{Q_{n}\right\}_{n \geq 0}$ is a periodic sequence. Hence, if we want to write several elements of this sequences, we have

$$
\frac{1}{2}(3-i-j-k),-\frac{1}{30}(1+9 i+7 j+17 k), \frac{1}{15}(10+i-2 k) .
$$

Therefore, one can use the elements of this new sequences to find the autocorrelation function. The autocorrelation function of a sequence is a measure of how different from the transformation of the given sequence[5]. The autocorrelation function has many important applications in engineering such as optical, coded diagramming.

Since the set of complex numbers is a special case of real quaternions, the special sequences defined on real quaternions can be thought of as a generalization of the sequences defined on complex numbers. So, one can use the elements of this new sequence as autocorrelation coefficients.

Now, let us consider a periodic autocorrelation function $C$ of the sequence $\left\{Q_{n}\right\}_{n \geq 0}[7]$.

$$
C(m)=\sum_{t=0}^{n-1} Q_{t} Q_{t+m}, \text { for } m \neq 0 \text { and } 1 \leq m \leq n-1
$$

Since the quaternion multiplication is not commutative, two alternative definitions of the autocorrelation function are possible. Let $C$ be the right periodic autocorrelation function of the sequence $\left\{Q_{n}\right\}_{n \geq 0}$, for $m \neq 0$ and for all $1 \leq m \leq n-1$, and define as

$$
C^{R}(m)=\frac{1}{\|Q\|} \sum_{t=0}^{n-1} Q_{t} \overline{Q_{t+m}}
$$

Then, let $C$ be the left periodic autocorrelation function of the sequence $\left\{Q_{n}\right\}_{n \geq 0}$, for $m \neq 0$ and $\forall 1 \leq m \leq n-1$, and define as

$$
C^{L}(m)=\frac{1}{\|Q\|} \sum_{t=0}^{n-1} \overline{Q_{t}} Q_{t+m}
$$

An alternative definition of the left autocorrelation function is given by the following formula;

$$
\operatorname{Alt}^{L}(m)=\frac{1}{\|Q\|} \sum_{t=0}^{n-1} \overline{Q_{t+m}} Q_{t}
$$

Since $A l t C^{L}(m)=\overline{C^{L}(m)}$, these definitions are equal to each other. In the following tables, for $\left\{Q_{n}\right\}_{n \geq 0}$ and $m=1,2,3,4$ we calculate the autocorrelation, right and left periodic autocorrelation values.

\begin{tabular}{|c|c|}
\hline$m$ & $C(m)$ \\
\hline 1 & $-9+3 k+\frac{1}{900}(3620+1676 i+1080 j+4208 k)$ \\
\hline 2 & $3+3 i+2 j+5 k+\frac{1}{30}(152-53 i-5 j-28 k)$ \\
\hline 3 & $2+i+2 j+3 k+\frac{1}{30}(182+4 i+42 j+4 k)$ \\
\hline 4 & $-9+2 i+2 j+k+\frac{1}{30}(123+13 i+127 j+119 k)$ \\
\hline
\end{tabular}


Table 2. The values $C(m)$

\begin{tabular}{|c|c|}
\hline$m$ & $C^{R}(m)$ \\
\hline 1 & $9+2 i+2 j+k+\frac{1}{900}(-1050+694 i+4070 j-3242 k)$ \\
\hline 2 & $-2-2 i+2 j+2 k+\frac{1}{30}(-56-18 j)$ \\
\hline 3 & $-2-i-2 j-3 k+\frac{1}{30}(-84+22 i+22 j+66 k)$ \\
\hline 4 & $9-2 i-2 j-k+\frac{1}{900}(-1050-694 i-4050 j-3242 k)$ \\
\hline
\end{tabular}

Table 3. The values $C^{R}(m)$

\begin{tabular}{|c|c|}
\hline$m$ & $C^{L}(m)$ \\
\hline 1 & $9-i-3 k+\frac{1}{30}(-37-73 i-47 j-163 k)$ \\
\hline 2 & $-2-2 i-2 j-3 k+\frac{1}{30}(-84+22 i+66 j+38 k)$ \\
\hline 3 & $-2+2 i+j+3 k+\frac{1}{30}(-84-2 i-66 j+22 k)$ \\
\hline 4 & $9+3 k+\frac{1}{30}(-35+73 i+47 j+141 k)$ \\
\hline
\end{tabular}

Table 4. The values $C^{L}(m)$

Some elements of the above tables $2,3,4$ can be calculate as below; by using the initial values, $Q_{0}=i+j+2 k, Q_{1}=$ $1+i+2 j+3 k$, the values $Q_{2}, Q_{3}, Q_{4}, Q_{5}, Q_{6}$ are as follows;

$$
Q_{2}=\frac{1}{2}(3-i-j-k), Q_{3}=-\frac{1}{30}(1+9 i+7 j+17 k), Q_{4}=\frac{1}{15}(10+i-2 k)
$$

and

$$
Q_{5}=i+j+2 k, \quad Q_{6}=1+i+2 j+3 k
$$

Thus,for $n=5$, this is a periodic sequence . Hence, we can write as

$$
\left\{i+j+2 k, 1+i+2 j+3 k, \frac{1}{2}(3-i-j-k),-\frac{1}{30}(1+9 i+7 j+17 k), \frac{1}{15}(10+i-2 k)\right\} .
$$

By the aid of the autocorrelation function, for the sequence $\left\{Q_{n}\right\}_{n \geq 0}$, we have the following sum.

$$
C(2)=\sum_{t=0}^{4} Q_{t} Q_{t+2}=3+3 i+2 j+5 k+\frac{1}{30}(152-53 i-5 j-28 k)
$$

Also, under favor of the right periodic autocorrelation function definition, for $m=4$, we have

$$
\begin{aligned}
& C^{R}(4)=\sum_{t=0}^{4} Q_{t} \overline{Q_{t+4}}=Q_{0} \overline{Q_{4}}+Q_{1} \overline{Q_{0}}+Q_{2} \overline{Q_{1}}+Q_{3} \overline{Q_{2}}+Q_{4} \overline{Q_{3}}, \\
& C^{R}(4)=9-2 i-2 j-k+\frac{1}{900}(-1050-694 i-4050 j-3242 k) .
\end{aligned}
$$

By using the definition of left periodic autocorrelation function, we can get the following formula.

$$
\begin{gathered}
C^{L}(3)=\sum_{t=0}^{4} \overline{Q_{t}} Q_{t+3}=\overline{Q_{0}} Q_{3}+\overline{Q_{1}} Q_{4}+\overline{Q_{2}} Q_{0}+\overline{Q_{3}} Q_{1}+\overline{Q_{4}} Q_{2}, \\
C^{L}(3)=-2+2 i+j+3 k+\frac{1}{30}(-84-2 i-66 j+22 k) .
\end{gathered}
$$


Now, we give the following theorem without proof.

THEOREM 1. For the Fibonacci quaternion sequence $\left\{Q_{n}\right\}_{n \geq 0}$, the set of right autocorrelation values is different from the set of left autocorrelation values.

THEOREM 2. For the Fibonacci quaternion sequence $\left\{Q_{n}\right\}_{n \geq 0}$ the following equalities are satisfied.

$$
\sum_{m=0}^{n-1}\left\|C^{L}(m)\right\|=\frac{1}{\|Q\|} \sum_{t_{1}=0}^{n-1} \sum_{t_{2}=0}^{n-1} \overline{Q_{t_{1}}}\left(C^{R}\left(t_{2}-t_{1}\right)\right) Q_{t_{2}}
$$

and

$$
\sum_{m=0}^{n-1}\left\|C^{R}(m)\right\|=\frac{1}{\|Q\|} \sum_{t_{1}=0}^{n-1} \sum_{t_{2}=0}^{n-1} Q_{t_{1}}\left(C^{L}\left(t_{2}-t_{1}\right)\right) \overline{Q_{t_{2}}} .
$$

Where $C^{R}(m)$ and $C^{L}(m)$ right and left periodic autocorrelation functions of the sequence $\left\{Q_{n}\right\}_{n \geq 0}$, respectively.

PROOF. From the definitions right and left autocorrelation function, we have

$$
\begin{aligned}
& \sum_{m=0}^{n-1}\left\|C^{L}(m)\right\|=\sum_{m=0}^{n-1}\left\|\frac{1}{\|Q\|} \sum_{t=0}^{n-1} \overline{Q_{t}} Q_{t+m}\right\| \\
& \sum_{m=0}^{n-1}\left\|C^{L}(m)\right\|=\left(\frac{1}{\|Q\|}\right)^{2} \sum_{m=0}^{n-1}\left(\left(\sum_{t_{1}=0}^{n-1} \overline{Q_{t_{1}}} Q_{t_{1}+m}\right)\left(\overline{\left.\sum_{t_{1}=0}^{n-1} \overline{Q_{t_{2}}} Q_{t_{2}+m}\right)}\right)\right. \\
& \sum_{m=0}^{n-1}\left\|C^{L}(m)\right\|=\frac{1}{\|Q\|^{2}} \sum_{m=0}^{n-1} \sum_{t_{1}=0}^{n-1} \sum_{t_{2}=0}^{n-1} \overline{Q_{t_{1}}} Q_{t_{1}+m} \overline{Q_{t_{2}+m}} Q_{t_{2}} \\
& \sum_{m=0}^{n-1}\left\|C^{L}(m)\right\|=\frac{1}{\|Q\|^{2}} \sum_{t_{1}=0}^{n-1} \sum_{t_{2}=0}^{n-1} \sum_{m=0}^{n-1} \overline{Q_{t_{1}}} Q_{t_{1}+m} \overline{Q_{t_{2}+m}} Q_{t_{2}} \\
& \sum_{m=0}^{n-1}\left\|C^{L}(m)\right\|=\frac{1}{\|Q\|^{2}} \sum_{t_{1}=0}^{n-1} \sum_{t_{2}=0}^{n-1} \overline{Q_{t_{1}}}\left(\sum_{m=0}^{n-1} Q_{t_{1}+m} \overline{Q_{t_{2}+m}} Q_{t_{2}}\right. \\
& \sum_{m=0}^{n-1}\left\|C^{L}(m)\right\|=\frac{1}{\|Q\|^{2}} \sum_{t_{1}=0}^{n-1} \sum_{t_{2}=0}^{n-1} \overline{Q_{t_{1}}}\left(\|Q\| C^{R}\left(t_{2}-t_{1}\right)\right) Q_{t_{2}} \\
& \left.\sum_{m=0}^{n-1}\left\|C^{L}(m)\right\|=\frac{1}{\|Q\|} \sum_{t_{1}=0}^{n-1} \sum_{t_{2}=0}^{n-1} \overline{Q_{t_{1}}}\left(C^{R}\left(t_{2}-t_{1}\right)\right) Q_{t_{2}}\right) .
\end{aligned}
$$

Thus, the equation (2.15) is true. Similarly, the equation (2.16) can be obtained. So, the proof is completed.

CONCLUSION. In this study, firstly Fibonacci quaternions were introduced by using Fibonacci numbers and some basic characteristics of it were given. Then, it was mentioned about that a special sequence was defined using the elements of the Fibonacci 
quaternion sequence with the help of the periodic sequence definition. And then, the autocorrelation values, right and left periodic autocorrelation values were calculated with the elements of the newly defined sequence. We would like to mention that autocorrelation functions for the Fibonacci quaternion sequences can be also calculated according to different conjugates.

\section{REFERENCES}

[1] Beth, T., Jungnickel D., and Lenz H. Design theory. Vol. 69. Cambridge University Press, 1999.

[2] Halici, S. On Fibonacci quaternions. Advances in Applied Clifford Algebras 22.2 (2012): 321-327.

[3] Hamilton, W.R. Ii on quaternions; or on a new system of imaginaries in algebra, Philosophical Magazine Series, Taylor \& Francis, 25:163, 10-13(1844).

[4] Horadam, A. F. Complex Fibonacci numbers and Fibonacci quaternions. The American Mathematical Monthly 70.3 (1963): 289-291.

[5] Jungnickel, D. and Pott, A. Perfect and almost perfect sequences. Discrete Applied Mathematics 95.1-3 (1999): 331359.

[6] Koshy, T. Fibonacci and Lucas Numbers with Applications. Wiley-Interscience, New York (2001).

[7] Kuznetsov, O. Perfect sequences over the real quaternions. Signal Design and its Applications in Communications, 2009. IWSDA'09. Fourth International Workshop on. IEEE, 2009.

[8] Luke, HD. Sequences and arrays with perfect periodic correlation. IEEE Transactions on Aerospace and Electronic Systems 24.3 (1988): 287-294.

[9] Iyer, M. R. A Note On Fibonacci Quaternions, The Fib. Quarterly, 3(1969), 225-229.

[10] Morais, JP., Georgiev, S. and Sprig, W. An Introduction to Quaternions. Real Quaternionic Calculus Handbook. Springer Basel, 1-34, 2014.

[11] Rouse Ball, W. W., and Coxeter, H. S. M. Mathematics recreation and essay. (1987).

[12] Vajda, S. Fibonacci and Lucas numbers, and the golden section: theory and applications. Courier Corporation, 1989. 